\title{
Wind tunnel testing of a generic telescope enclosure
}

\section{Tait S. Pottebaum, Douglas G. MacMynowski}

Tait S. Pottebaum, Douglas G. MacMynowski, "Wind tunnel testing of a generic telescope enclosure," Proc. SPIE 5495, Astronomical Structures and Mechanisms Technology, (29 September 2004); doi: 10.1117/12.550798

EDent: SPIE Astronomical Telescopes + Instrumentation, 2004, Glasgow, United Kingdom 


\title{
Wind tunnel testing of a generic telescope enclosure
}

\author{
Tait S. Pottebaum ${ }^{* a}$ and Douglas G. MacMynowski ${ }^{\mathrm{b}}$ \\ ${ }^{a}$ Graduate Aeronautical Laboratories; ${ }^{b}$ Control and Dynamical Systems \\ California Institute of Technology, 1200 E. California Blvd., Pasadena, CA, USA, 91125
}

\begin{abstract}
The design of future large optical telescopes must take into account the wind-induced buffeting of the telescope structure caused by large-scale flow structures and turbulence inside the dome. However, estimating the resulting degradation in image quality is difficult due to our relatively poor understanding of the flow inside the dome. Data has been collected in a scaled wind-tunnel test of a telescope enclosure to understand the flow-field around the region near the dome opening where the secondary mirror and supporting structure would be subjected to wind loads. Digital particle image velocimetry (DPIV) data was collected in a vertical plane near the dome opening to obtain mean velocity and fluctuation kinetic energy. In addition, hotwire data was collected along the telescope axis to obtain temporal spectra of the velocity, and flow visualization was used to determine the general flow patterns. In addition to its direct use in telescope modeling and design, this data is of particular value in validation of computational fluid dynamic (CFD) analyses, so that CFD can be used with confidence in future design work.
\end{abstract}

Keywords: Extremely-Large-Telescopes, Wind-buffeting, wind tunnel, DPIV

\section{INTRODUCTION}

Various design studies are currently underway for the next generation of large ( $>20 \mathrm{~m}$ diameter) ground-based optical telescopes, such as TMT, GMT, and Euro50. Several studies have analyzed the wind-induced buffeting of the telescope structure that results from turbulence inside the telescope enclosure $e^{1-3}$ and concluded that this vibration must be taken into account in the design process, affecting the design of the telescope enclosure, the structure and the control system. However, estimating the degradation in image quality that results from the wind-induced vibration is difficult due to our relatively poor understanding of the wind inside the enclosure.

Wind loads are relevant both over the primary mirror (M1) and on the secondary mirror (M2) and nearby supporting structure. Although the cross-sectional area of M2 is small compared to that of M1, the structure around the mirror is exposed to much higher wind loads. Steady forces can be compensated for by active control of the telescope structure. Estimates of the unsteady forces, however, require knowledge of the unsteady turbulent wind velocity. The resulting optical consequences can then be computed from an integrated model that predicts the actively controlled structural response $e^{2-4}$.

An understanding of the wind inside a telescope enclosure can be obtained from various sources, including measurements in observatories ${ }^{5}$, computational fluid dynamics (CFD) $)^{6,7}$ or through wind- or water-tunnel experiments . $^{8}$ In addition to its direct use in telescope modeling and design, experimental data is of particular value in validation of CFD analyses, so that CFD can be used with confidence in future design work.

For an $80 \mathrm{~m}$ diameter telescope dome in a $12 \mathrm{~ms}^{-1}$ external wind (roughly the 85 th percentile wind on Mauna Kea), the full-scale Reynolds number of the flow is well above $10^{7}$, so the full-scale flow in the telescope enclosure is definitely turbulent. To be meaningful, the experimental Reynolds numbers must therefore be sufficiently high that the flow is also turbulent. Of particular importance is the flow field around M2. For design purposes, it is essential to understand not only the wind speed or pressure at a few isolated locations, but more details of the flow pattern that can be used, for example, to help choose the clearance between M2 and the dome.

Data has been collected in a scaled wind-tunnel test of a telescope enclosure to understand the flow-field around the region near the dome opening where M2 and its supporting structure would be subjected to wind loads. The model was

* tpotteba@ caltech.edu; phone 1626395 4462; fax 16265775258 
intended to be representative of a generic empty telescope dome at approximately $1 \%$ of the expected full-scale enclosure size for a $30 \mathrm{~m}$ diameter telescope. While the elevation angle of the dome opening was fixed at $30^{\circ}$ from zenith, any azimuth angle could be tested. Digital particle image velocimetry (DPIV) data was collected in a vertical plane near the dome opening to obtain mean velocity and fluctuation kinetic energy information. In addition, hotwire data was collected along the telescope axis to obtain temporal spectra of the velocity. Flow visualization was used to determine the general flow patterns in the enclosure. DPIV data was collected only for azimuth angles of $0^{\circ}$ and $180^{\circ}$ with respect to the wind so that the mean flow along the centerline remained planar due to symmetry.

\section{SCALING}

Several important parameters must be scaled properly in order for this set of experiments to be related to a full-scale telescope. In particular, the Reynolds number of the flow and the scaling of frequencies must be considered.

The Reynolds number is defined as

$$
\operatorname{Re}=\frac{\mathrm{U}_{\infty} \Lambda}{\mathrm{v}}=\frac{\mathrm{U}_{\infty} \mathrm{L}}{\mathrm{v}},
$$

where $U_{\infty}$ is the freestream velocity, $v$ is the kinematic viscosity, and $\Lambda$ is the characteristic length scale, which in this case is taken to be the aperture dimension, $\mathrm{L}$, which equals the square root of the aperture area. This ratio represents the relative importance of inertial forces to viscous forces in the momentum balance for a flow. High Reynolds number flows are typically turbulent, though the critical Reynolds number for turbulence depends on the type of flow.

There are two main types of frequencies that can appear in this type of flow. First, there may be frequencies that are caused by the convection of periodically occurring large scale structures in the flow. Second, there may be acoustic resonances that result in periodic flow.

For convective frequencies, a characteristic frequency can be defined as

$$
\mathrm{f}_{0}=\frac{\mathrm{U}_{\infty}}{\Lambda}=\frac{\mathrm{U}_{\infty}}{\mathrm{L}} \text {. }
$$

It is not possible to consider how the length scale affects the characteristic frequency without also considering the influence of the velocity scale. If the freestream velocity is held constant, then $\mathrm{f}_{0}$ is proportional to $\mathrm{L}^{-1}$. For a given enclosure geometry $\mathrm{L} / \mathrm{R}$ is constant, so $\mathrm{f}_{0}$ will scale as $\mathrm{R}^{-1}$. If, however, Reynolds number is held constant, $\mathrm{U}_{\infty}$ is proportional to $\mathrm{L}^{-1}$. This leads to $\mathrm{f}_{0}$ scaling as $\mathrm{R}^{-2}$. These scaling arguments assume that the kinematic viscosity is the same in the experiments as at the telescope site.

Acoustic resonances can also result in frequencies appearing in the flow field. The relevant resonance for a telescope enclosure with a single opening for viewing is the Helmholtz resonance in the limit with the neck length going to zero. For this type of resonance, the frequency will be

$$
\mathrm{f}_{\mathrm{H}}=\frac{\mathrm{c}}{2 \pi} \sqrt{\frac{\mathrm{L}}{\mathrm{V}}},
$$

where $\mathrm{c}$ is the speed of sound and $\mathrm{V}$ is the enclosed volume. For a given enclosure design $\mathrm{V}$ will be proportional to $\mathrm{R}^{3}$, so $f_{H}$ will be proportional to $R^{-1}$. The sound speed affects this frequency as well, so there is a small dependence on the altitude at which the telescope is located. In an experiment where the Reynolds number is matched to the full scale Reynolds number, $\mathrm{f}_{\mathrm{H}} / \mathrm{f}_{0}$ will scale as $\mathrm{R}$, assuming that the sound speed is constant. In an experiment where the freestream Mach number $\left(\mathrm{M}=\mathrm{U}_{\infty} / \mathrm{c}\right)$ is matched to the full scale Mach number, $\mathrm{f}_{\mathrm{H}} / \mathrm{f}_{0}$ will be independent of $\mathrm{R}$. If the enclosure radius is fixed and the velocity is varied in a set of experiments with constant sound speed, $\mathrm{f}_{\mathrm{H}} / \mathrm{f}_{0}$ will scale as $\mathrm{U}_{\infty}^{-1}$.

\section{METHODOLOGY}

\subsection{Test facility}

These experiments were carried out in the Lucas Adaptive Wall Wind Tunnel (AWT). This facility uses adaptive wall technology in the test section to reduce the wall effects typical of straight-wall wind tunnel tests. While the tunnel is 
operating, pressure measurements are taken along the length of the test section floor and ceiling. A one-step predictive algorithm combines the pressure measurements with knowledge of the current wall contour to determine the new wall contour that will result in the wall following a streamline for the current model configuration. The test section ceiling and floor are then deformed to the desired contour. The system effectively "tricks" the air into thinking it is in an infinite flow field, rather than confined by the walls of the tunnel. For the purpose of this study, the adaptive walls allow for a larger model, relative to the test section cross-section, than a conventional rigid-walled wind tunnel.

The test section of the Lucas AWT is $7.62 \mathrm{~m}$ long and has a rectangular cross-section $1.83 \mathrm{~m}$ wide and $1.52 \mathrm{~m}$ high when un-adapted. The ceiling and floor each have a maximum displacement of $0.30 \mathrm{~m}$ away from the mid-plane of the tunnel. A rigid ground plane equipped with a yaw table was installed in the test section to support the ELT enclosure model. With the ground plane installed, the test section floor is used to control the location of the leading edge stagnation point on the ground plane, and only the ceiling is used to adapt for the model. Over a wide range of flow speeds, the required wall deflection at each streamwise location is nearly equal to the cross-sectional area of the model at that location. Therefore, the maximum allowable model cross-sectional area was $0.56 \mathrm{~m}^{2}$.

\subsection{Enclosure model}

All of the extremely large telescope initiatives are still in their early stages, so no specific sites have been selected and no specific point designs have been identified as primary candidates. In light of this uncertainty, we have modeled airflow through a "generic" large telescope enclosure with a very simple geometry. The "full scale" enclosure is taken to consist of a $90 \mathrm{~m}$ diameter hemisphere placed upon a $30 \mathrm{~m}$ tall right cylinder with the same diameter. Inside the enclosure is a floor that is placed $10 \mathrm{~m}$ above the base of the cylinder. All walls have a thickness of $1 \mathrm{~m}$, and the enclosure aperture is modeled as a square opening whose sides measure $35 \mathrm{~m}$. There is no additional venting of the enclosure.

The wind tunnel model of the ELT enclosure consisted of a $0.83 \mathrm{~m}$ diameter hemisphere of clear acrylic plastic mounted on a $0.28 \mathrm{~m}$ tall right cylinder with the same diameter made of white acrylic plastic. The cylinder portion of the model had a wall thickness of $9.5 \mathrm{~mm}$. The hemisphere portion wall thickness varied due to the manufacturing process, with a maximum thickness of $9 \mathrm{~mm}$ where it attached to the cylinder and a minimum thickness of $5 \mathrm{~mm}$ at the zenith. A square with side length $0.32 \mathrm{~m}$ was removed from the hemisphere at an elevation of $30^{\circ}$ from zenith. A $0.09 \mathrm{~m}$ thick cylindrical wooden base, with diameter matching the interior diameter of the acrylic cylinder, was attached to the center of the ground plane yaw table using four $1 / 2$ " bolts. The acrylic model was placed over the wooden base and attached to it using twelve 1/4"-20 machine screws. The azimuth angle of the model aperture was varied by rotating the yaw table, with $0^{\circ}$ azimuth corresponding to the aperture facing directly into the wind. The enclosure is empty because preliminary telescope designs have not yet been determined.

\subsection{Experimental techniques}

\subsubsection{Flow visualization}

Flow patterns can be visualized in a wind tunnel through many different methods. For this experiment, smoke visualization and tufts were used.

Smoke visualization works by injecting smoke into the flow at a known location and observing how the smoke particles are convected. Individual images of the smoke are obtained by flashing a light source at known times and recording images. This produces qualitative information about the large scale structures in the flow and the general circulation pattern of the flow.

Tufts are used to determine the direction and steadiness of flow near a surface. Tufts of string are attached to the surface, and the shear stress produced by the flow orients the tufts in the direction of the flow. A qualitative sense of the flow's unsteadiness can be determined by observing whether the tufts vibrate or remain pointed in one direction. 


\subsubsection{Hot-wire anemometry}

A hot-wire anemometer measures fluid velocity by sensing changes in heat transfer from a small, electrically-heated sensor (hot-wire) exposed to the flow. The hot-wire sensor is maintained at a constant temperature using a feedback control circuit, as shown in Figure 1. The hot-wire's resistance is a function of temperature, so the control circuit actually works to maintain a constant resistance across the hot-wire. The flow past the hot-wire cools it, and this heat loss is compensated for by the current through the hot-wire probe. The magnitude of the current needed to keep the temperature constant is directly related to heat transfer and, thus, flow velocity. The output voltage of the control circuit, which is proportional to the current, is recorded as a time series during an experiment and later converted into a velocity time series using calibration data. Hot-wires are particularly useful for measurements of unsteady velocities due to the high frequency response that can be obtained.

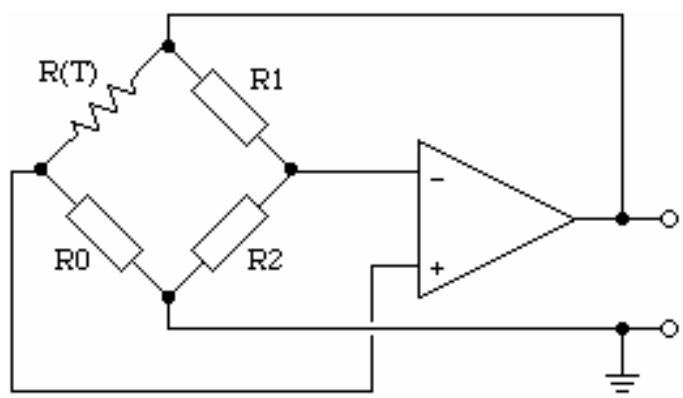

Figure 1: Hot-wire anemometer control circuit. The hot-wire probe resistance is represented by $R(T)$. The circuit uses a Wheatstone Bridge and an operational-amplifier to maintain $R(T) /[R(T)+R 0]=R 1 /[R 1+R 2]$.

\subsubsection{Digital particle image velocimetry}

Digital particle image velocimetry (DPIV) is a technique for determining the in-plane velocity field in a cross-section of a flow. It is the digital version of the particle image velocimetry (PIV) technique and was first used by Willert and Gharib ${ }^{9}$. Experiments are typically carried out by seeding the flow with reflective or fluorescent particles, illuminating a thin cross-section of the flow field with pulsed monochromatic light, and capturing images of the particles using a CCD video camera. Displacement estimates are obtained by locating the peak of the cross-correlation of the intensity between small interrogation windows within pairs of consecutive images. This method of processing depends on the particles within the interrogation window primarily being convected by the flow, rather than large velocity differences existing within the window, so that a large correlation peak exists. The displacements are then converted to velocities using the known time separation between pulses of the light source. Many refinements of the original DPIV processing technique have been developed. In particular, window-shifting reduces the effect of particles entering and leaving an interrogation window between images by relocating the interrogation window in the second image based upon an initial displacement estimate without window-shifting. A variety of different processing techniques are currently in use, each with its own strengths and weaknesses ${ }^{10}$.

\subsection{Experimental setup}

A high-resolution CCD video camera (model CV-M4+CL; JAI PULNiX Inc., Sunnyvale, CA, USA) with an 8mm focal length lens was positioned inside the model along with a mirror in order to image a region of the vertical plane on the model centerline containing the enclosure aperture. The center of the top of the field-of-view was tangent to the removed portion of the hemisphere at the middle of the aperture. A physical area of approximately $0.38 \mathrm{~m}$ by $0.30 \mathrm{~m}$ was imaged onto 1280 by 1024 pixels. In addition to the imaging equipment, a hot-wire probe (model 1210; TSI Incorporated, Shoreview, MN, USA) was mounted on a motorized traverse inside of the model. The traverse was aligned with the model such that the probe could be moved along the nominal telescope axis (i.e., the line connecting the spherical center of the hemisphere and the center of the aperture). For all azimuth angles, the hot-wire probe was oriented to measure the magnitude of the velocity in the vertical plane containing the nominal telescope axis. In addition, at $90^{\circ}$ azimuth the probe was oriented to measure velocities in the plane normal to the telescope axis. The sensing element of the hot-wire probe was a cylindrical element $1.3 \mathrm{~mm}$ in length and $3.2 \mu \mathrm{m}$ in diameter. The positions of the camera and mirror, the camera field-of-view, and the mounting base for the hot-wire traverse are shown in Figure 2. Cabling for the camera, hot-wire and traverse exited the model through a hole in the wooden base and an opening in 
the yaw table. The cables then exited the wind tunnel through the pressure matching gap at the downstream end of the test section.

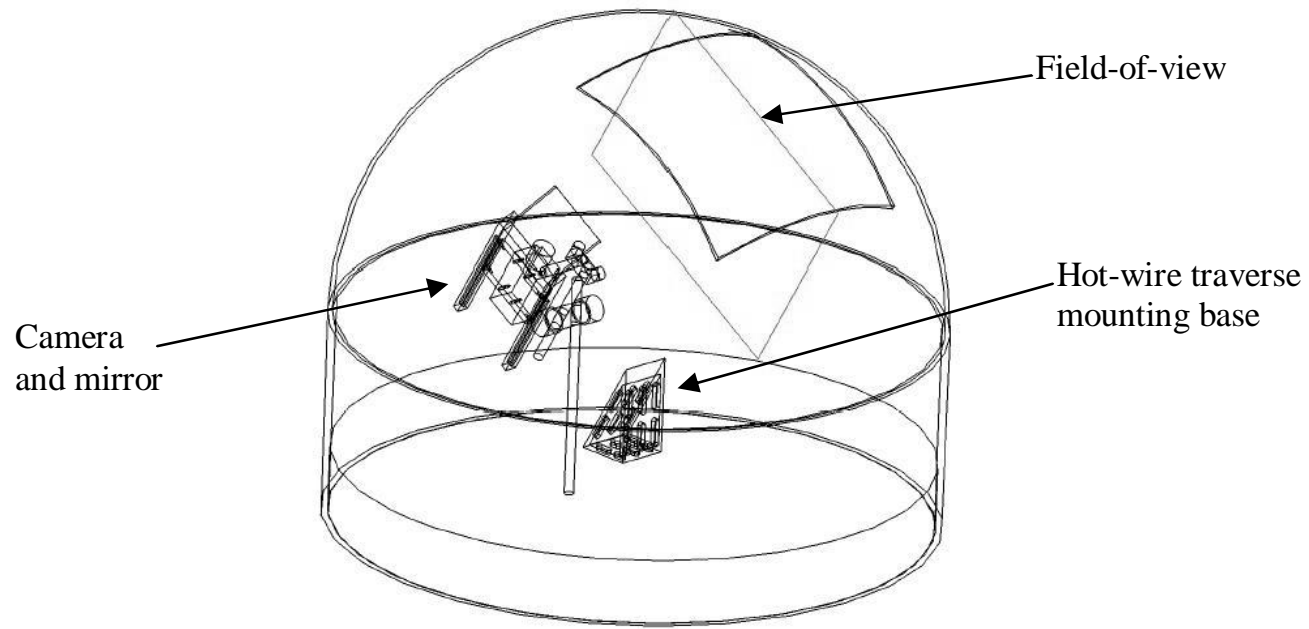

Figure 2: Positions of the camera, mirror, hot-wire traverse mounting base, and camera field-of-view inside of the wind tunnel model

For DPIV and smoke visualization, a pair of frequency doubled Nd:YAG lasers (Gemini-PIV; New Wave Research Inc., Fremont, CA, USA) were used to generate a vertical light sheet along the centerline of the test section. The laser heads were located outside of the test section, and the light entered the test section though an approximately $10 \mathrm{~mm}$ diameter hole in the side wall. Beam turning and sheet forming optics were suspended from a streamlined crossbar mounted to the two test section side walls near the ceiling. The optical elements and their associated translation and rotation stages were housed inside a streamlined fairing with openings to allow the laser beam to enter and the laser sheet to exit.

Smoke for flow visualization was produced using a smoke wand located upstream of the enclosure model. Water droplets were used as the seeding particles for DPIV. The droplets were produced by four fuel-injection type nozzles connected to the building water supply. Three of the nozzles were located inside of the enclosure model. The fourth nozzle was located on the upstream exterior surface of the model, just above the point on the centerline where the hemisphere and cylinder sections are joined.

DPIV images were recorded directly to computer hard disk. Laser pulse timing was controlled using a delay generator (model DG535; Stanford Research Systems, Sunnyvale, CA, USA) triggered by the camera exposure enabled output. The hot-wire probe was operated using a Matilda anemometer (GALCIT), with the output signal recorded to computer through an analog-to-digital converter.

\subsection{Experimental conditions and procedures}

For this experiment, the wind tunnel was primarily operated at a freestream speed of $35 \mathrm{~ms}^{-1}$ in order to achieve the highest possible Reynolds number without causing damage to the model. This speed was reproduced between separate runs to within $0.05 \mathrm{~ms}^{-1}$. Additional tests were carried out at $20 \mathrm{~ms}^{-1}$ and $28 \mathrm{~ms}^{-1}$. The wind tunnel test section ceiling was adapted to the model shape before beginning data collection, and was left at that shape throughout the experiment. The air temperature in the wind tunnel was typically $22^{\circ} \mathrm{C}$, and the freestream pressure was $101 \mathrm{KPa}$. The freestream turbulence intensity in the wind tunnel has not been measured, but is estimated to be approximately $1 \%$ of the freestream velocity.

Smoke visualization was carried out for $0^{\circ}$ and $180^{\circ}$ azimuth angles at $35 \mathrm{~ms}^{-1}$. The smoke was illuminated with a pulsed sheet of laser light along the centerline of the tunnel. Images were recorded using the camera inside of the enclosure and a tripod mounted video camera outside of the wind tunnel. The smoke visualization images were used to 
examine large scale structures in the flow near the aperture. In addition, tufts were used for the $0^{\circ}$ and $180^{\circ}$ azimuth angle cases. Strips of tufts were located at four locations on the interior of the cylindrical section of the enclosure and at four locations on the raised floor. Handheld video camera footage was taken of the tuft and was used to determine the general flow pattern in the enclosure.

Hot-wire data was acquired for enclosure azimuth angles between $0^{\circ}$ and $180^{\circ}$ in $30^{\circ}$ increments at $35 \mathrm{~ms}^{-1}$. Additional hot-wire data was acquired for $0^{\circ}$ and $90^{\circ}$ azimuth angles at $20 \mathrm{~ms}^{-1}$ and $28 \mathrm{~ms}^{-1}$. Data was collected along the nominal telescope axis at 20 equally spaced locations between $45 \%$ and $103 \%$ of the hemisphere's radius. At each location, the control circuit output voltage was recorded for $3 \mathrm{~s}$ at a $30 \mathrm{KHz}$ sampling rate. The power spectral density (PSD) of each time series was computed using Welch's modified periodogram method with a Hanning window of size 16384 samples and no overlap. Due to a faulty electrical connection discovered after data was acquired, only the PSD, and not mean velocity, is available from the hot-wire data.

DPIV data was acquired for the $0^{\circ}$ and $180^{\circ}$ azimuth cases at $35 \mathrm{~ms}^{-1}$. Before acquiring DPIV data, the hot-wire probe and traverse were removed from the model to eliminate a source of reflections. DPIV data was collected in a series of short runs. This was necessary because water droplets collected on the mirror and camera lens, resulting in degraded image quality. Between runs, the mirror, camera lens, and model were dried using towels. Each run consisted of 200 images for the $0^{\circ}$ case and 1000 images for the $180^{\circ}$ case. Images were digitally recorded at a rate of 24 frames per second with a pixel depth of 10-bits. Due to the large difference in velocities inside of the model and outside of the model, it was not possible to select a pulse separation time that resulted in optimal particle displacements in all portions of the field-of-view. The time between laser pulses within an image pair was $200 \mu$ s for the $0^{\circ}$ case and $300 \mu$ s for the $180^{\circ}$ case in order to obtain reasonable results throughout the field-of-view. A total of 2400 pairs of images were obtained and processed to generate velocity field estimates for the $0^{\circ}$ case. For the $180^{\circ}$ case, 4495 pairs of images were obtained and processed.

Before extracting velocity field measurements from the DPIV data, the images were preprocessed. First, the two least significant bits in the recorded images were dropped, resulting in 8-bit images compatible with existing processing software. Then, optical distortion introduced by the short focal length camera lens was removed using a mapping obtained from calibration images of a regular grid of points. Next, stationary features in the images, such as reflections from the model surface, were identified by forming averages of all of the data images. Two average images were formed for each case, one from all of the "even" frames and the other from all of the "odd" frames. This was done in order to account for possible differences in the illumination between "even" and "odd" frames. The large number of individual images in the averages guaranteed that only persistent, stationary features exist in the average images. The appropriate average image was then subtracted from each data image.

Velocity field data was extracted from the preprocessed images using 64-by-64 pixel interrogation windows with $75 \%$ overlap of adjacent windows for both cases. The large window size was necessary due to the large displacements in the region of flow outside of the model. Displacements were first estimated by cross-correlating the same region in both images of a pair. After removing outliers based upon velocity magnitude and the overlap of adjacent interrogation windows, this displacement field was used to shift the interrogation window in the second image for use in a second cross-correlation. Outliers were removed from this second displacement field. The vector field was then smoothed to be consistent with the window overlap, and the displacements were converted to velocities. For the $180^{\circ}$ case, an additional processing step was included to refine the velocity estimates to 32-by-32 pixel interrogation windows with $50 \%$ overlap.

\section{RESULTS}

\subsection{Flow visualization}

At $0^{\circ}$ azimuth, smoke visualization captures the repetitive roll-up of the shear layer over the aperture into large, unsteady vortices. These vortices travel into the enclosure. At other times, the shear layer is deflected upwards away from the enclosure. Observation of the surface tufts at this azimuth angle indicate that the general flow inside the enclosure is down along the downstream surface, upstream and slightly upwards along the enclosure sides, and upward 
on the upstream surface. While the interior flow has some unsteadiness, there did not appear to be flow reversals. These results are summarized in Figure 3A.

For the $180^{\circ}$ case, the smoke visualization did not reveal any large scale structures near the aperture. Based upon observation of the surface tufts, the general flow inside the enclosure is down along the upstream surface, downstream and slightly upwards along the enclosure sides, and approximately stagnant on the downstream surface. The level of unsteadiness inside of the enclosure is significantly lower for the $180^{\circ}$ case than for the $0^{\circ}$ case. These results are summarized in Figure 3B.

A

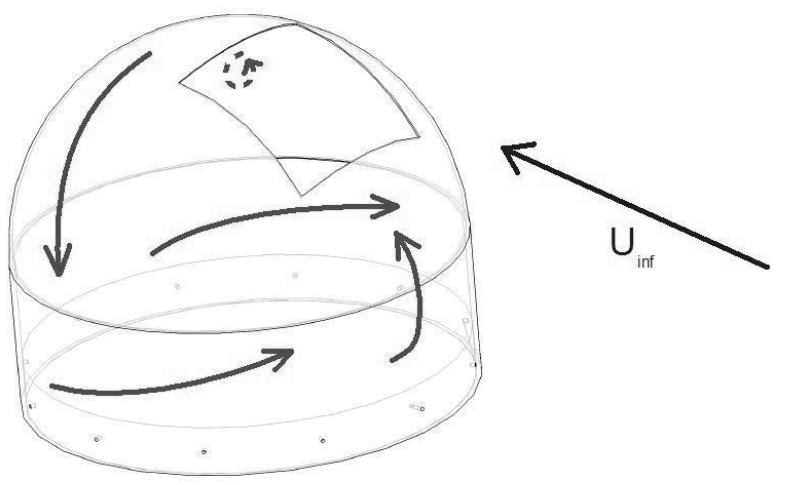

$\mathrm{B}$

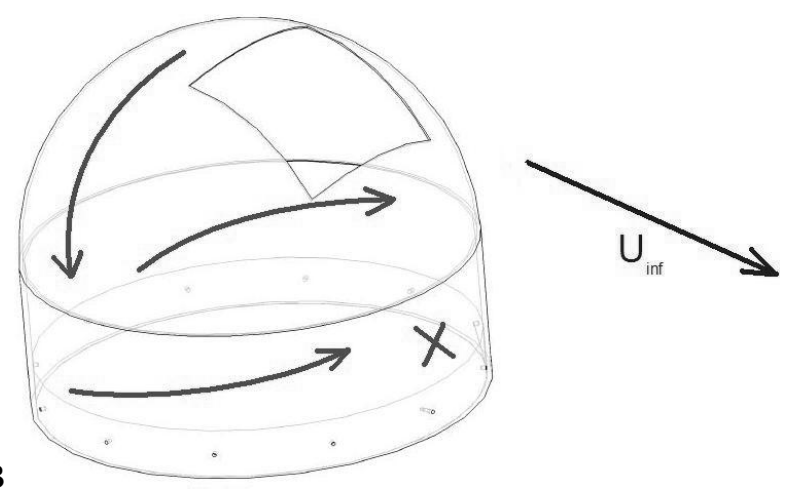

Figure 3: General circulation for (A) $0^{\circ}$ and (B) $180^{\circ}$ azimuth.

\subsection{Hot-wire anemometry}

For azimuth angles $\leq 90^{\circ}$, the PSD of the hot-wire data includes distinct peaks above the background level at multiples of approximately $0.6 \mathrm{f}_{0}$. An example of this type of data set is shown in Figure 4A. For azimuth angles $>90^{\circ}$, there are no such peaks in the PSD plots. Figure 4B shows a data set typical of this condition. For all azimuth angles, a portion of the PSD shows a -5/3 roll-off typical of turbulent flow. At high frequencies, the PSD flattens out, and in some cases additional peaks in the PSD are observed.

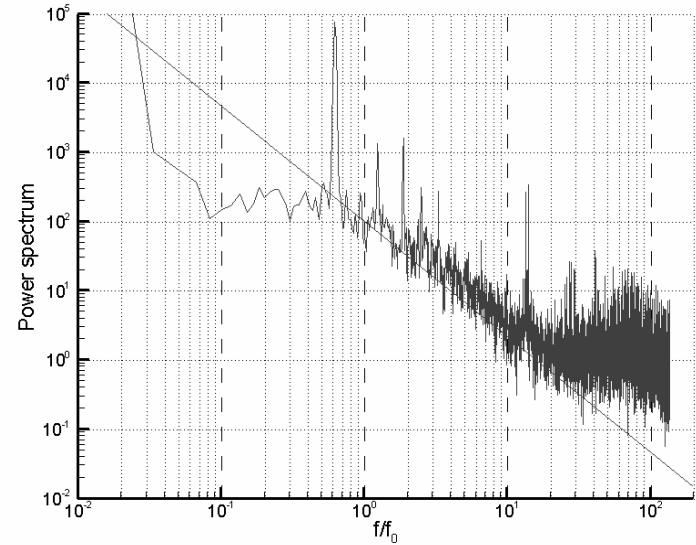

A

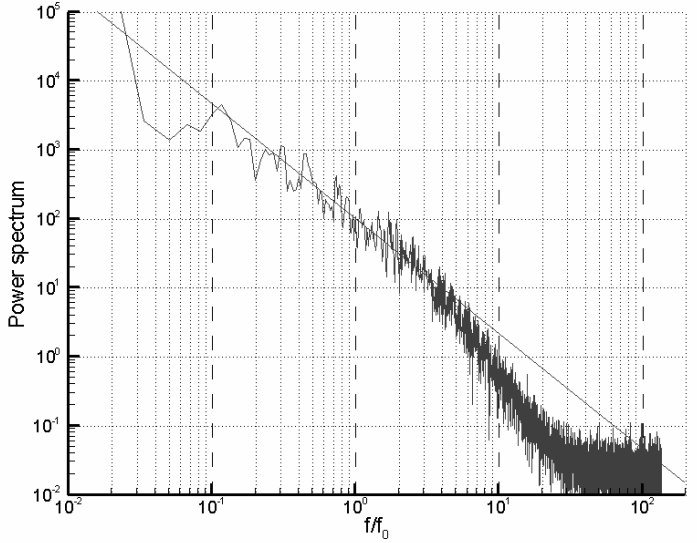

B

Figure 4: Power spectral density (in arbitrary units) for $(\mathrm{A}) \mathrm{O}^{\circ}$ and $(\mathrm{B}) \mathbf{1 8 0}^{\circ}$ azimuth at $35 \mathrm{~ms}^{-1}$. $\mathrm{Hot}_{-w i r e}$ position is $93 \%$ of the enclosure radius along the telescope axis. The straight line with negative slope in each plot is a $-5 / 3$ power law.

The relative magnitudes of the frequency peaks depend upon the freestream velocity. This can be seen by comparing Figure 4A, Figure 5A, and Figure 5B. Each of these PSD plots represents data taken at the same location and at the same azimuth angle, but at different freestream velocities. The non-dimensional frequencies at which the peaks occur does not appear to depend on the freestream velocity. This is shown in Figure 6, which plots the non-dimensional frequencies of the first two peaks in the PSD for each combination of azimuth angle and flow speed tested. 


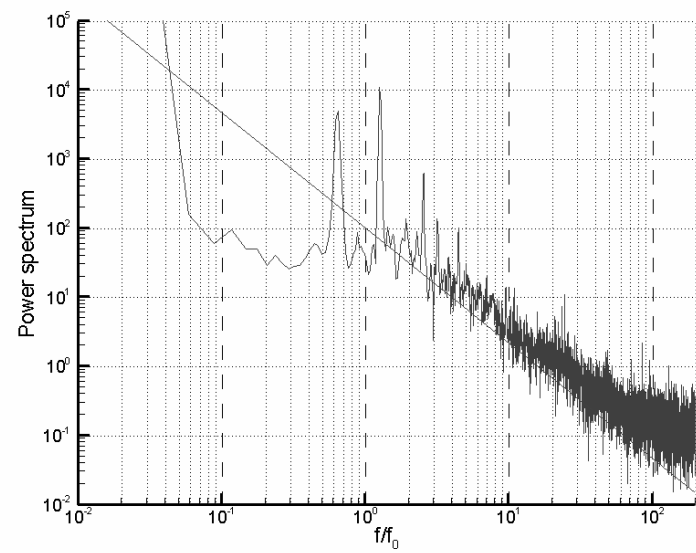

A

Figure 5: Power spectral density (in arbitrary units) for $0^{\circ}$ azimuth at (A) $20 \mathrm{~ms}^{-1}$ and (B) $28 \mathrm{~ms}^{-1}$. Hot-wire position is $93 \%$ of the enclosure radius along the telescope axis. The straight line with negative slope in each plot is a $-5 / 3$ power law.

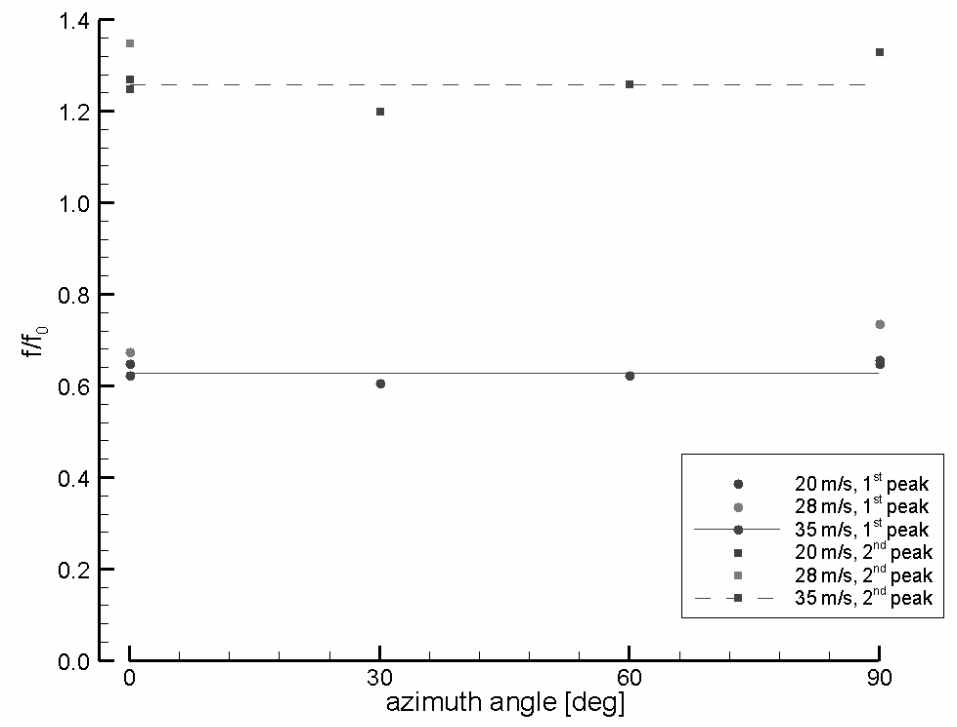

Figure 6: Summary of frequencies observed in hot-wire signals for various azimuth angles and flow speeds. All data is with the hot-wire located at $93 \%$ of the enclosure radius along the telescope axis.

\subsection{DPIV}

The mean and root-mean-square (rms) in-plane flow fields measured using DPIV for $0^{\circ}$ azimuth are shown in Figure 7. The origin of the coordinate system in these plots is the spherical center of the hemisphere. A shear layer exists across the aperture and becomes very thick near the downstream edge of the opening. All of the visible flow is directed generally downstream. The rms levels are highest in the shear layer, and the elevated rms levels extend significantly into the enclosure on the downstream half of the opening. A region of upward directed flow with slightly elevated rms levels exists near the bottom of the field of view $(x / R \sim-0.2, y / R \sim 0.4)$.

The same quantities are plotted for $180^{\circ}$ azimuth angle in Figure 8. Here, the flow is all generally directed upstream. There is a local maxima in mean velocity magnitude located inside of the enclosure. While both the mean and rms velocity magnitudes are generally lower than in the $0^{\circ}$ case, the rms levels fall off much more gradually inside of the 
enclosure for the $180^{\circ}$ case. A region of upward directed flow with slightly elevated rms levels again exists near the bottom of the field of view $(\mathrm{x} / \mathrm{R} \sim 0.2, \mathrm{y} / \mathrm{R} \sim 0.4)$.
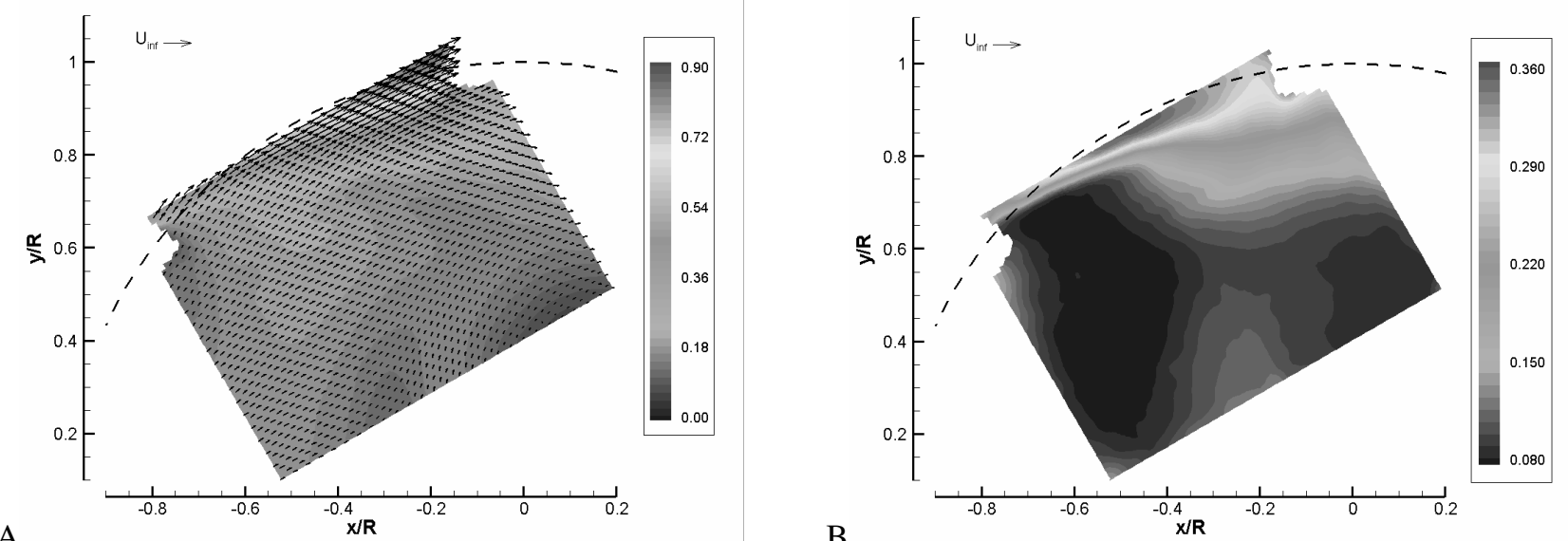

Figure 7: Normalized (A) mean and (B) rms flow fields near the aperture for $0^{\circ}$ azimuth at $35 \mathrm{~ms}^{-1}$. Velocities are scaled by the freestream velocity $\left(U_{\text {inf }}\right)$.
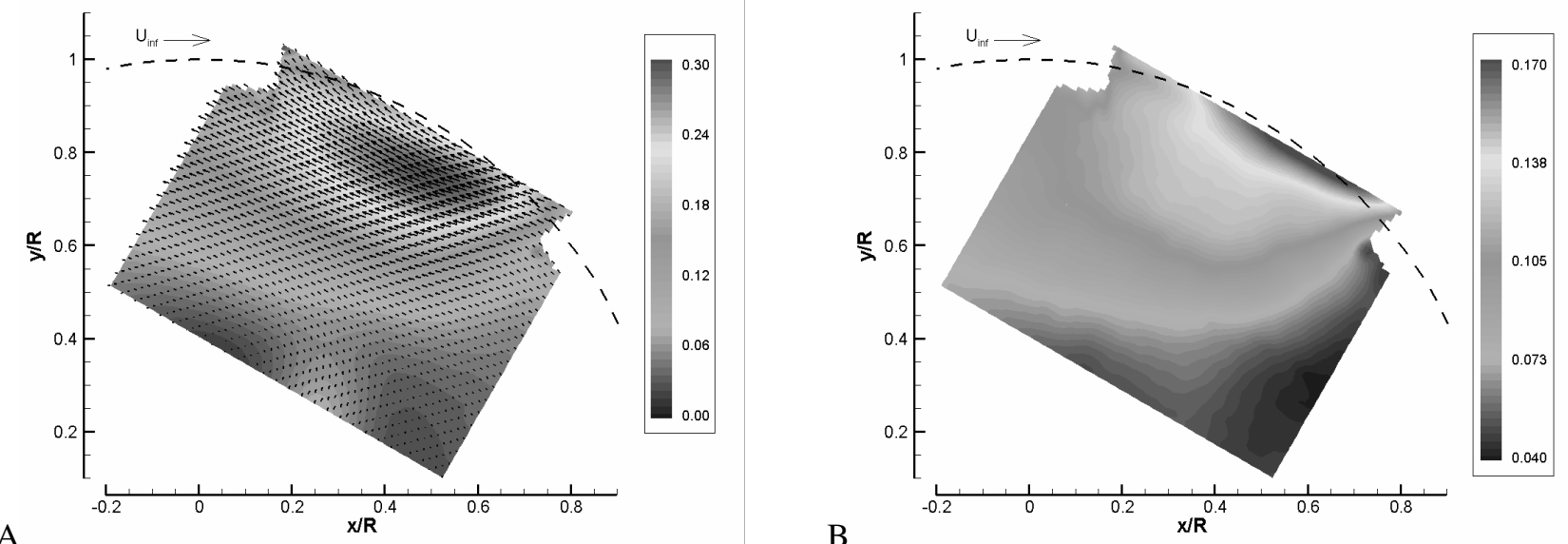

Figure 8: Normalized (A) mean and (B) rms flow fields near the aperture for $180^{\circ}$ azimuth at $35 \mathrm{~ms}^{-1}$. Velocities are scaled by the freestream velocity $\left(\mathrm{U}_{\mathrm{inf}}\right)$.

\section{DISCUSSION}

\subsection{Upwind viewing}

For the $0^{\circ}$ azimuth angle case at $35 \mathrm{~ms}^{-1}$, the smoke visualization clearly indicates the repetitive roll-up of the aperture shear layer into large individual vortices. The hot-wire data for this same case demonstrates that this is a periodic rollup, with a frequency of approximately $0.6 \mathrm{f}_{0}$. For lower flow speeds, there is comparable or greater power in the peak around $1.2 \mathrm{f}_{0}$. This indicates that a different roll-up process may be occurring.

For a frequency in the flow due to the convection of periodic structures, the ratio $\mathrm{f} / \mathrm{f}_{0}$ is related to the convection speed $\mathrm{f}$ the structures and the length scale of the structures. Assuming that a fluid structure convects with a velocity $\mathrm{U}_{\mathrm{c}}$ and that the spacing between structures is such that there are $n$ structures in the distance $\mathrm{L}$, where $\mathrm{n}$ is a whole number, the temporal frequency appearing in the flow will be

$$
\mathrm{f}=\frac{\mathrm{nU}_{\mathrm{c}}}{\mathrm{L}} .
$$


The ratio of the frequency to the characteristic frequency is therefore

$$
\frac{\mathrm{f}}{\mathrm{f}_{0}}=\mathrm{n} \frac{\mathrm{U}_{\mathrm{c}}}{\mathrm{U}_{\infty}},
$$

representing whole number multiples of the fraction of the freestream speed at which the structures convect. By applying this analysis to the hot-wire data summarized in Figure 6, it becomes evident that vortices in the aperture shear layer are convected at a speed of about $0.6 \mathrm{U}_{\infty}$. This is consistent over the range of azimuth angles for which peaks in the PSD were observed.

In addition, the relative magnitudes of the peaks in the PSD indicate that at different speeds, the number of vortices spanning the enclosure may vary. For example, at $20 \mathrm{~ms}^{-1}$ the $n=2$ peak is larger than the $n=1$ peak. This may indicate that the shear layer is rolling-up into a vortex twice in the time it takes a vortex to convect across the opening. This would result in two vortices being present in the opening at the same time.

The selection of the roll-up mode number, $\mathrm{n}$, is believed to be dependent on the Helmholtz frequency of the enclosure. At the different speeds tested in this experiment, the ratio $\mathrm{f}_{\mathrm{H}} / \mathrm{f}_{0}$ takes on different values, as listed in Table 1. For the two higher speed cases, $\mathrm{f}_{\mathrm{H}} / \mathrm{f}_{0}$ is close to the value corresponding to $\mathrm{n}=1$, and the first peak in the PSD is dominant (Figure $4 \mathrm{~A}$ and Figure $5 \mathrm{~B}$ ). For the lowest speed, $\mathrm{f}_{\mathrm{H}} / \mathrm{f}_{0}$ lies between the frequencies corresponding to $\mathrm{n}=1$ and $\mathrm{n}=2$, but is closer to the $n=2$ frequency. In this case, the second peak in the PSD is slightly larger than the first peak (Figure 5A). This suggests that the Helmholtz frequency is determining the frequency at which the shear layer is actually rolling-up from among the various frequencies compatible with the convection velocity. For the full scale enclosure in $12 \mathrm{~ms}^{-1}$ winds, $\mathrm{f}_{\mathrm{H}} / \mathrm{f}_{0}$ would be approximately 1.7 , so one would expect to see an $\mathrm{n}=3$ type roll-up of the shear layer. At lower wind speeds, $\mathrm{f}_{\mathrm{H}} / \mathrm{f}_{0}$ would be even larger, so higher modes would be expected.

Table 1: Dependence of non-dimensional Helmholtz frequency on freestream velocity

\begin{tabular}{cc}
$\mathbf{U}_{\infty}\left[\mathbf{m s}^{-1}\right]$ & $\mathbf{f}_{\mathbf{H}} / \mathbf{f}_{\mathbf{0}}$ \\
\hline 20 & 0.99 \\
28 & 0.71 \\
35 & 0.57
\end{tabular}

The mean and rms velocity fields observed with DPIV for $0^{\circ}$ azimuth angle are consistent with the roll-up of vortices evident in the flow visualization and hot-wire data. The shear layer appears thick in the mean flow and the rms magnitude is large deep inside the dome on the downstream side of the opening due to the formation of the large vortices.

For determining the effects on $\mathrm{M} 2$, it is useful to consider the mean and rms in-plane velocity magnitude profiles along the telescope axis. This is extracted from the DPIV data and shown in Figure 9A for the $0^{\circ}$ azimuth case. Both the mean and rms magnitudes are relatively constant deep inside the enclosure, but increase sharply in the shear layer at about $0.8 \mathrm{R}$. This suggests that it may be beneficial to design an enclosure that results in M2 being located deep enough inside that dome that it is not sitting in the shear layer. With this in mind, it is important to note that as the mode number $\mathrm{n}$ increases, the radius of the vortices should decrease. The depth into the dome which the region of high mean velocities and high rms magnitudes extends will therefore be smaller for lower flow speeds typical of the full scale enclosure.

The region of upward flow at the bottom of the DPIV field-of-view is believed to be due to the triangular base for the hot-wire traverse. The general flow inside of the enclosure hits this ramp and is directed upwards. This portion of the flow field is likely to be entirely different in an enclosure containing a telescope.

\subsection{Downwind viewing}

For the $180^{\circ}$ azimuth case, the flow field is significantly different from the cases previously discussed. The flow in the upstream direction evident in both the flow visualization and the DPIV data indicates that, for this case, the aperture sits inside the region of reversed flow in the enclosure's wake. There is no strong shear layer over the aperture, and therefore no significant peaks in the PSD of the hot-wire signals. 
For determining the effects of the flow on $\mathrm{M} 2$, it is again useful to consider the velocity magnitude profiles along the telescope axis. This data is plotted for the $180^{\circ}$ azimuth case in Figure 9B. While the maximum mean and rms velocity magnitudes are smaller for this case than for the $0^{\circ}$ case, high levels of fluctuations actually extend deeper into the enclosure. This makes it much more difficult to determine a desirable location for M2 based only upon this simple metric. The local maximum in mean velocity magnitude is due to the change in flow direction between the wake region and the outer flow-there is a shear layer located above the enclosure across which the flow reverses direction, so the mean velocity magnitude must go to zero somewhere in that shear layer.

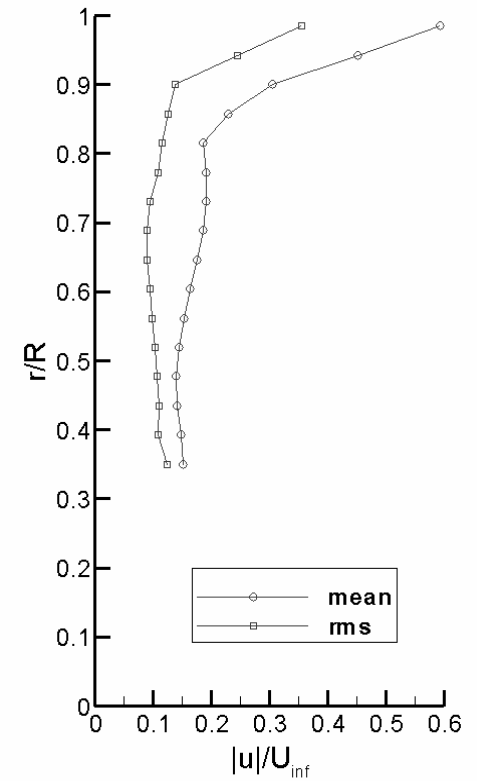

A

Figure 9: Normalized mean and rms in-plane velocity magnitudes on the telescope axis for (A) $0^{\circ}$ and $(B) 180^{\circ}$ at $35 \mathrm{~ms}^{-1}$.

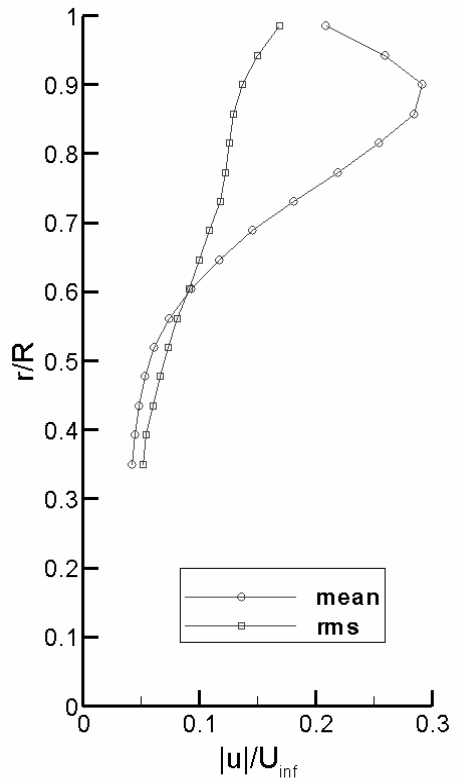

B

\section{CONCLUSIONS}

Two very different types of flow inside of a telescope enclosure exist depending on the azimuth angle of the aperture with respect to the wind.

For upwind viewing, there is a strong shear layer over the telescope aperture. This shear layer drives the flow inside of the enclosure. The shear layer rolls-up periodically into vortices which can extend inside of the enclosure and may produce significant unsteady forces on the secondary mirror. The frequency at which the shear layer roles up is well described by a convective velocity, determined to be about $0.6 \mathrm{U}_{\infty}$, and a mode number corresponding to the number of vortices spanning the aperture at one time. The particular mode that is excited is determined by the Helmholtz resonance. From the current data, it cannot be determined how additional features of a full scale enclosure, such as mitigation efforts or venting, will affect the shear layer roll-up.

For downwind viewing, the aperture is located inside the wake of the enclosure. This results in reversed flow in the wake driving the flow inside the enclosure. In this configuration, there are no significant large scale unsteady flow structures to produce forces on the telescope. However, the turbulence level in the flow is significant and can produce forces on the telescope.

The data obtained from this test are being used to validate CFD studies of airflow in telescope enclosures ${ }^{11}$ and to develop models of the wind loads for use in telescope structural designs ${ }^{12}$. 
Additional experiments have recently been conducted to explore the effects of venting and of different flow speeds on the observed large scale structures and general circulation patterns. Analysis of the data from these new experiments is ongoing.

\section{ACKNOWLEDGEMENTS}

The Thirty Meter Telescope (TMT) Project is a partnership of the Association of Universities for Research in Astronomy (AURA), the Association of Canadian Universities for Research in Astronomy (ACURA), the California Institute of Technology and the University of California. The partners gratefully acknowledge the support of the Gordon and Betty Moore Foundation, the US National Science Foundation, the National Research Council of Canada, the Natural Sciences and Engineering Research Council of Canada, and the Gemini Partnership.

Mory Gharib (Caltech) provided substantial guidance and technical expertise in the design of this experiment and the application of the DPIV technique. The Lucas AWT staff (Christopher Krok and Jerry Landry) supplied technical assistance in the areas of model construction, flow visualization, troubleshooting and test documentation. Joeleff Fitzsimmions (HIA) and Konstantinos Vogiatzis (AURA NIO) also provided troubleshooting assistance and consulted on the selection of test conditions.

\section{REFERENCES}

1. S. Padin, W. Davison,"Model of image degredation due to wind buffeting on an extremely large telescope," Applied Optics, 43, 592-600, 2004.

2. G. Angeli, A. Segurson, R. Upton, B. Gregory, M. Cho, "Integrated modeling tools for large ground based optical telescopes," Proceedings of the SPIE, 5178, 49-63, 2003.

3. D.G. MacMartin, "Control Challenges for Extremely Large Telescopes," Smart Structures and Materials 2003: Industrial and Commercial Applications of Smart Structures Technology, E.H. Anderson (Ed.), 2003.

4. G.Z. Angeli, S. Roberts, D. MacMynowski, J. Fitzsimmions, J. Dunn, K. Vogiatzis, A. Sheinis, "Modelling tools to estimate the performance of the Thirty Meter Telescope: an integrated approach," Proceedings of the SPIE, 5497, 2004.

5. M.K. Cho, L.M. Stepp, G.Z. Angeli, D.R. Smith, "Wind loading of large telescopes," Large Ground-Based Telescopes, Oschmann, Stepp (Eds.), 352-367, 2002.

6. D.S. DeYoung,"Numerical Simulations of Airflow in Telescope Enclosures," The Astronomical Journal, 112, 2896-2908, 1996.

7. K. Vogiatzis, D. DeYoung, "Numerical Simulations of Flow in Extremely Large Telescope Enclosures," SPIE Workshop on Extremely Large Telescopes, 2003.

8. T. Kiceniuk, K. Potter, "Internal air flow patterns for the Keck 10 meter telescope observatory dome," California Institute of Technology, Keck Observatory Report \#166, 1986.

9. C.E. Willert, M. Gharib,"Digital Particle Image Velocimetry," Experiments in Fluids, 10, 181-193, 1991.

10. S.P. McKenna, W.R. McGillis,"Performance of digital image velocimetry processing techniques," Experiments in Fluids, 32, 106-115, 2002.

11. K. Vogiatzis, A. Segurson, G.Z. Angeli, "Estimating the effect of wind loading on extremely large telescope performance using computational fluid dynamics," Proceedings of the SPIE, 5497, 2004.

12. D.G. MacMynowski, G. Angeli, K. Vogiatzis, J. Fitzsimmons, S. Padin, "Parametric modeling and control of telescope wind-induced vibration," Proceedings of the SPIE, 5497, 2004. 